\title{
Force-Controlled MEMS Rotary Microgripper
}

\author{
Busara Piriyanont, Student Member, IEEE, Anthony George Fowler, Member, IEEE, \\ and S. O. Reza Moheimani, Fellow, IEEE
}

\begin{abstract}
This paper presents a force-controlled microelectromechanical systems rotary microgripper with integrated electrothermal sensors. The proposed microgripper achieves a large displacement $(85 \mu \mathrm{m})$ at low driving voltages $(\leq 80 \mathrm{~V})$ Closed-loop force control is implemented to ensure the safety of the operation where the controller gain is experimentally tuned so that the desired response is achieved. One of the main contributions of this work is the implementation of a nulldisplacement feedback control force-sensing technique, where the controller counteracts the input disturbance (contact force) and an integrated electrothermal displacement sensor provides a feedback signal to close the control loop. In this manner, the contact force is measured without moving the structure. Finally, the effectiveness of the controller and the performance of the proposed microgripper are verified by a set of experiments. The results demonstrate the satisfactory performance of the proposed forcecontrolled microgripper in a practical application. [2014-0374]
\end{abstract}

Index Terms-MEMS micro-gripper, micromanipulation, gripping force, force control, electrothermal sensor.

\section{INTRODUCTION}

A MEMS MICRO-GRIPPER is a microelectromechanical device consisting of actuators, sensors, and other mechanical elements, capable of performing manipulation tasks on micron-size objects $(1-100 \mu \mathrm{m})$. The micro-gripper is an important tool in micromanipulation, with typical applications involving micro-assembling, testing microsystem components, measuring mechanical properties [1], and handling/manipulating biological cells and tissues [2].

A variety of micro-grippers have been proposed based on different fabrication processes, actuation methods and forcesensing principles [3]-[8]. One example is reported in [9], which presents a monolithically fabricated micro-gripper with a capacitive force sensor. The device achieves a relatively large output force and displacement at the tip of the gripping arm. However, a significant driving voltage is required $(150 \mathrm{~V})$, and a relatively large proportion of the device's area is occupied by the comb-drive actuator. Even though the device's capacitive sensor offers a high sensing bandwidth, it has low resolution and sensitivity. Alternatively, an electrothermally actuated micro-gripper in [10] achieves a large output force/displacement at a much lower driving voltage.

Manuscript received December 8, 2014; accepted December 31, 2014. Date of publication January 21, 2015; date of current version July 29, 2015. Subject Editor E.-S. Kim.

The authors are with the School of Electrical Engineering and Computer Science, University of Newcastle, Callaghan, NSW 2308, Australia (e-mail: piriyanont@hotmail.com; anthony.fowler@uon.edu.au; reza.moheimani@newcastle.edu.au).

Color versions of one or more of the figures in this paper are available online at http://ieeexplore.ieee.org.

Digital Object Identifier 10.1109/JMEMS.2015.2388539
However, due to the high operating temperature $\left(105^{\circ} \mathrm{C}\right)$ of the electrothermally actuated gripper, it requires high power consumption and exhibits the buckling effect. Also, the high temperature of the device during operation is undesirable for bio-manipulation where the properties of the sample and/or the liquid medium are concerned. Another design discussed in [11] is a piezoelectric driven micro-gripper with strain gauges for force measurement. Although such a device achieves a large output force/displacement using a bridgetype displacement amplifier with a rotary bearing mechanism, undesirable phenomena including hysteresis nonlinearity and creep are observed. The hysteresis nonlinearity significantly reduces the precision of the actuator. Therefore, piezoelectric actuators commonly require a control method to improve the resolution and precision of the gripping operation. The major advantages of using strain gauges for force sensing are their simplicity, acceptable accuracy, speed and low costs. Nonetheless, this approach is limited by its ability to resolve the gripping force $(\mathrm{mN})$ with sufficient precision, given that many microassembly tasks require a higher force resolution $(\mu \mathrm{N})$.

In order to provide an effective tool for micromanipulation tasks, a force sensor and closed-loop control implementation are needed to ensure that the force applied by the microgripper does not damage the captured sample. A variety of force sensors and control strategies for micro-grippers have been proposed within the literature. Force control based on position control using visual feedback was reported in [12] and [13]. The visually-based feedback control method relies on a sensory capability which is based on proprioceptive sensors. The control schemes that employ proprioceptive sensors may have poor performance because of changes in the environment and/or modeling errors. Therefore, visuallybased position control is not sufficient to maintain the applied gripping force in a safe range. Force and position controllers are implemented for a hybrid thermo-piezoelectric microgripper in [14]. The controller operates in two modes, where it automatically switches between coarse and fine position modes. Due to a lack of integrated sensors, external optical displacement sensors are used to measure the deflection of the gripping arms and estimate the gripping force. Although the feedback system is reported to track the desired set points, a time delay and slow response are observed during the coarse positioning mode. Direct control of the gripping force in the range of milli to micro newtons has been achieved in [15]-[17]. The micro-grippers developed in [18] and [19] use only one arm for force sensing and control. In [20], a voice coil motor (VCM) is used for actuation of a micro-gripper, with strain gauge sensors being used to implement position and 
TABLE I

A Comparison of the Attributes of EXISTING MiCro-GripPer Designs

\begin{tabular}{|l|l|c|c|c|c|}
\hline Actuation & Force sensing & Driving voltage(V) & Displacement $(\mu \mathrm{m})$ & Sensitivity & Reference \\
\hline \hline Electrothermal & Piezoresistive & 4.5 & 32 & $0.588 \mathrm{NV}^{-1}$ & {$[4]$} \\
Electrothermal & none & 2 & 11 & - & {$[21]$} \\
Electrostatic & none & 55 & 22 & - & {$[22]$} \\
Electrostatic & Capacitive & 150 & 100 & $1.818 \mathrm{mNV}^{-1}$ & {$[9]$} \\
Electrostatic & Electrothermal & 80 & 90 & $1.95 \mu \mathrm{NV}^{-1}$ & This work \\
\hline
\end{tabular}

force control. Even though a VCM offers high force values, the results show that both controlled position and force outputs are considerably noisy.

In this paper, a force-controlled MEMS micro-gripper with integrated electrothermal sensors is presented. This work builds on the previous initial characterization results reported in [25]. The proposed force control is implemented to ensure that the gripping force is maintained at the chosen setpoint, therefore preventing delicate samples from being damaged during the micromanipulation operation. Also, this work employs a null-displacement feedback control force sensing technique which eliminates the difficulty dealing with the nonlinearity in microstructures at large displacement. In addition, a force calibration technique is presented where the sensitivity of the force sensor is determined using capacitive and angular displacement measurements. The performance of the presented MEMS rotary micro-gripper is compared with other existing micro-gripper designs as summarized in Tab. I.

The paper is organized as follows. Section II outlines the micro-gripper's working principle and characterization of the device. The actuators' electrostatic nonlinearity and the characteristics of the electrothermal sensor are investigated in this section. Section III provides the system identification and experimental force calibration. A feedback controlled sensing technique and a force control scheme is developed in Section IV. Next, experimental studies on force tracking and micro-bead gripping are carried out in Section $\mathrm{V}$ to verify the effectiveness of the force-controlled micro-gripper. Finally, Section VI concludes this paper.

\section{PRinciple of MEMS Rotary MiCRO-GRIPPER}

\section{A. Working Principle}

The gripper consists of a pair of gripping arms with an initial opening of $100 \mu \mathrm{m}$, and two sets of electrothermal displacement sensors and rotary comb-drives. Each gripping arm connects to actuators and heat sink plates via a curved shuttle, and is suspended by a series of flexures. The schematic structure of the MEMS rotary micro-gripper is illustrated in Fig. 1. The electrothermal displacement sensor is incorporated into the gripping arm via the shuttle, where the sensor consists of two resistive heaters and a heat sink positioned adjacent to the resistive heaters with a $3 \mu \mathrm{m}$ gap. At rest, the sensors $\left(R_{1}, R_{2}\right)$ are biased at the selected operating voltage, with the heat generated from each heater conducting across the air gap to the heat sink attached to the rotary shuttle. In the micro-gripper's neutral position, the overlap between the heat sink and each beam heater is equal, resulting

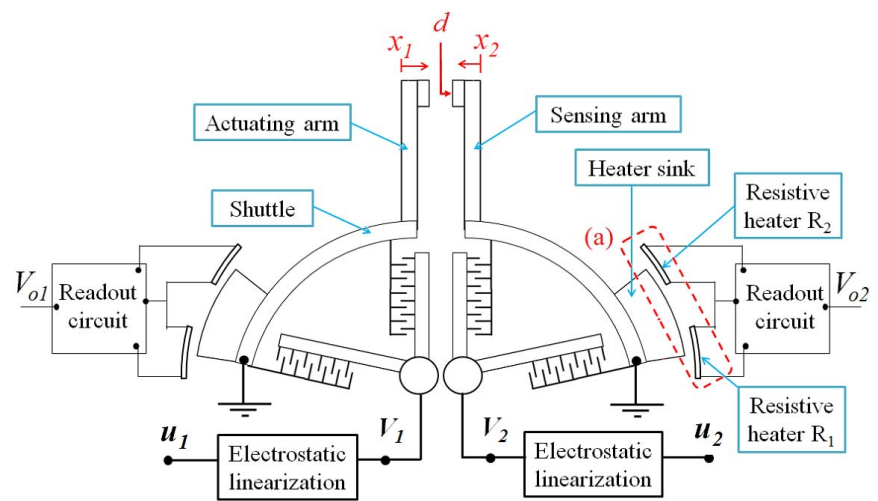

Fig. 1. The schematic of the MEMS rotary micro-gripper with (a) integrated electrothermal sensor. A square root function applied to the actuating signal is used to cancel the nonlinearity in the electrostatic actuator.

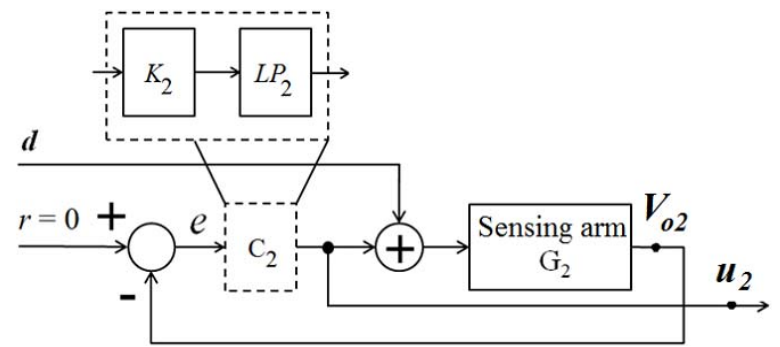

Fig. 2. Block diagram of the null displacement force feedback control, where the input to the system is the disturbance force $d$, and the output of the system is the control signal $u_{b}$.

in the heaters having identical temperatures and resistances. Once the sensing arm experiences a contact force, the heat sink (which is connected to the gripping arm) is pushed toward the right, as indicated in Fig. 3. The deviation of the heat sink's position induces a difference in the heat fluxes from the two beams, creating differential changes in their temperature. Due to the temperature-dependent resistivity of silicon, these temperature changes result in differential resistance variations, which are converted into output voltages via the use of a half Wheatstone bridge and an instrumentation amplifier.

In this work, we employ a null-displacment feedback control force sensing technique, where the contact force is measured at the sensing arm's null position, as reported in [24]-[26]. Due to the fact that the force being measured is no longer dependent on the mass and stiffness of the sensing structure, it eliminates the difficulty in dealing with the nonlinearity of the mechanical structure at large displacements. The null displacment forcesensing method is based on feedback control as illustrated in Fig. 2, where the contact force is treated as an input 

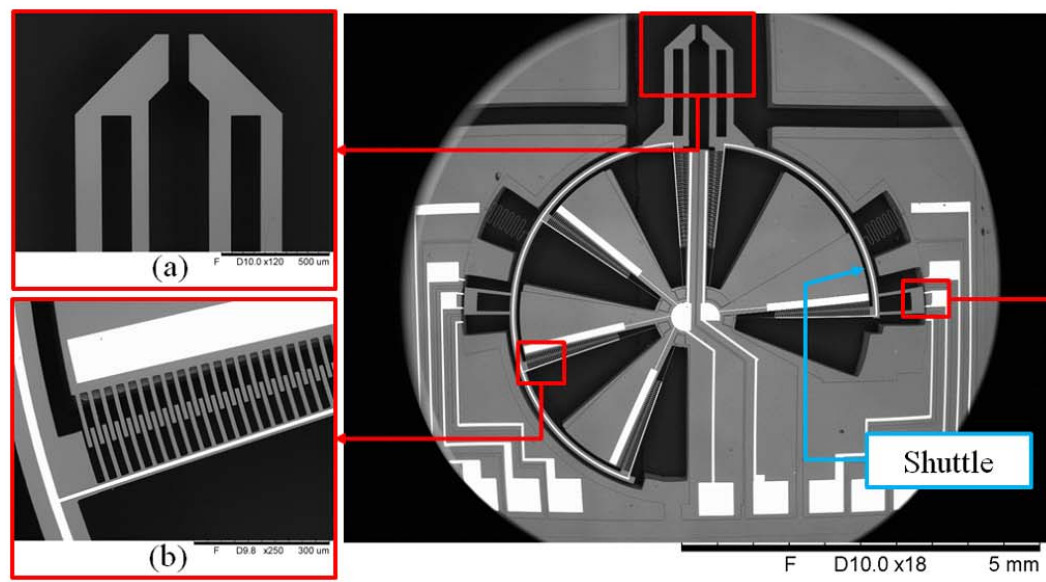

(a)

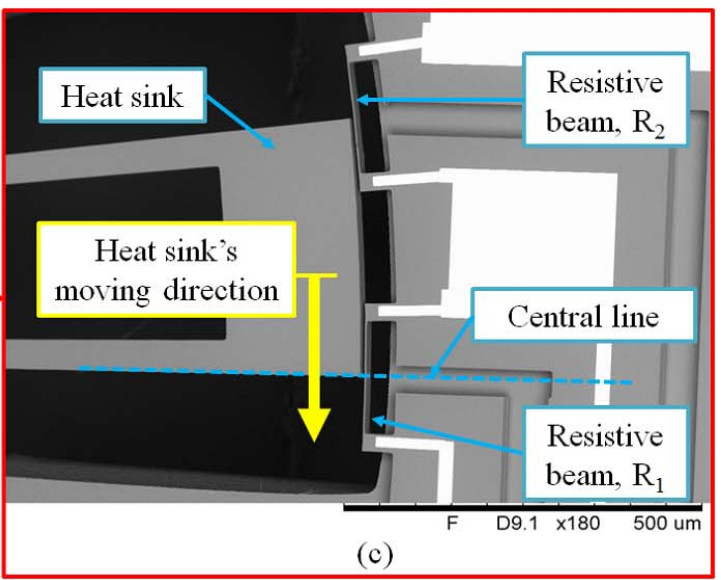

(c)

Fig. 3. An image of the MEMS rotary micro-gripper with zoomed-in images showing a section of (a) the gripping arms, (b) the rotary comb-drive, and (c) electrothermal displacement sensor.

disturbance $d$ and the output of the system is the control signal that is used to compensate for the disturbance. With the set-point of the controller being set to the sensing arm's equilibrium position $(r=0)$, the controller $C_{2}$ generates the required control signal to counteract the disturbance, therefore restoring the displacement of the sensing arm to zero. Note that the controller gain $\left(K_{2}\right)$ is experimentally tuned and selected to be 150 , where the system achieves satisfactory force tracking and disturbance rejection. At this point, the electrostatic force generated due to the control signal is equivalent to that of the disturbance force (contact force), thus the contact force can be measured by monitoring and interpreting the control signal $u_{b}$. The empirical relationship between the contact force and the control signal is experimentally calibrated and described later in Section III.

\section{B. Device Analysis and Characterization}

The fabricated device is an electrostatic-based microgripper, using a rotary comb-drive to generate torsional motion (small angle) where the output displacement is amplified by the length of the gripping arm. The rotary comb-drive is a capacitive actuator that consists of two interdigitated curved finger structures where one set of comb fingers is fixed while the other set is suspended by flexures, allowing the structure to move. The total capacitance [27] of the structure can be calculated from (1),

$$
C_{t o t}=\epsilon_{0}\left(\theta+\theta_{o}\right) h\left\{\sum_{i=1}^{n-1}\left[\ln \left(a_{i}\right)\right]^{-1}+\sum_{i=0}^{n-1}\left[\ln \left(b_{i}\right)\right]^{-1}\right\}
$$

where $a_{i}=\frac{R_{0}+2 i\left(W_{f}+g\right)}{R_{0}+2 i\left(W_{f}+g\right)-g}, b_{i}=\frac{R_{0}+(2 i+1)\left(W_{f}+g\right)}{R_{0}+2 i\left(W_{f}+g\right)+W_{f}}, \epsilon_{0}$ is the permittivity of free space $\left(8.85 \times 10^{-12} \mathrm{Fm}^{-1}\right), \theta$ is the overlapping angle, $\theta_{o}$ is the initial engagement angle, $h$ is the thickness of the comb fingers, $R_{0}$ is the radius of the first comb finger, $i$ refers to the $i^{\text {th }}$ comb finger, $W_{f}$ is the finger width, $g$ is the gap between adjacent fingers, and $n$ is the number of combs. An attractive electrostatic force is created when
TABLE II

Rotary COMb-Drive Design PARAMETERS

\begin{tabular}{ccc}
\hline \hline Parameter & Description & value (unit) \\
\hline$n_{1}, n_{2}$ & Number of comb fingers & 200,100 \\
$h$ & Device thickness & $25(\mu \mathrm{m})$ \\
$g$ & Comb-fingers gap & $4(\mu \mathrm{m})$ \\
$R_{o}$ & Radius of first comb finger & $600(\mu \mathrm{m})$ \\
$W_{f}$ & Comb finger width & $10(\mu \mathrm{m})$ \\
$\theta_{o}$ & Initial engagement angle & $1\left(^{\circ}\right)$
\end{tabular}

a voltage is applied across the comb structure, causing the comb fingers to be drawn together. The generated torque [27] is proportional to the square of the driving voltage and a differential change of capacitance as expressed in (2), and is described in its expanded form in (3).

$$
\begin{aligned}
\tau & =\frac{1}{2}\left(\frac{\partial C}{\partial \theta}\right) V^{2} \\
\tau & =\frac{1}{2} \epsilon_{0} h\left\{\sum_{i=1}^{n-1}\left[\ln \left(a_{i}\right)\right]^{-1}+\sum_{i=0}^{n-1}\left[\ln \left(b_{i}\right)\right]^{-1}\right\} V^{2}=\beta V^{2}
\end{aligned}
$$

For simplicity, $\beta$ is assigned to represent the electrostatic constant of the comb-drive as described in (4)

$$
\beta=\frac{1}{2} \epsilon_{0} h\left\{\sum_{i=1}^{n-1}\left[\ln \left(a_{i}\right)\right]^{-1}+\sum_{i=0}^{n-1}\left[\ln \left(b_{i}\right)\right]^{-1}\right\}
$$

Using the design parameter values given in Tab. II, the electrostatic constant of the actuating comb-drive $\left(\beta_{1}\right)$ and sensing comb-drive $\left(\beta_{2}\right)$ are determined to be $14.1478 \mathrm{pNmV}^{-2}$ and $7.0739 \mathrm{pNmV}^{-2}$ respectively.

To obtain the static characteristic of the comb-drive, the tip displacement is measured using a Polytec Micro System Analyzer (MSA-400), where the fitted quadratic function of the measured tip displacement is described as (5):

$$
x_{1}=0.013 \times V_{1}^{2}, \quad x_{2}=0.0078 \times V_{2}^{2}
$$


The device achieves a large output displacement $(85 \mu \mathrm{m})$ at a comb-drive voltage of $80 \mathrm{~V}$. The quadratic relationship between comb-drive voltage and electrostatic force/torque contributes to a static nonlinearity in the system $\left(\tau \propto V^{2}\right)$. As depicted in Fig. 1, the nonlinearity of the comb-drive is cancelled out by cascading the actuator with the inverse function of its quadratic behavior, where the inverse function outputs the comb-drive voltage $V^{2}$ by performing a square root operation on the control signal $u$ as given in (6a) and (6b). This results in a one-to-one relationship between control signal $u$ and sensor output $V_{o}$.

$$
\begin{aligned}
& V_{1}=33.71 \sqrt{u_{1}} \\
& V_{2}=32.11 \sqrt{u_{2}}
\end{aligned}
$$

By substituting (6a) and (6b) into (3), the electrostatic torque and force of the actuating comb-drive $\left(\tau_{1}, F_{1}\right)$ and sensing comb-drive $\left(\tau_{2}, F_{2}\right)$ can be expressed in terms of control signal $u$ as shown in (7a)-(7d).

$$
\begin{aligned}
\tau_{1} & =1,136 \beta_{1} u_{1}=\left(16.077 \times 10^{-9}\right) u_{1} \\
F_{1} & =\frac{\tau_{1}}{l}=\left(4.03 \times 10^{-6}\right) u_{1} \\
\tau_{2} & =1,030 \beta_{2} u_{2}=\left(7.2927 \times 10^{-9}\right) u_{2} \\
F_{2} & =\frac{\tau_{2}}{l}=\left(1.87 \times 10^{-6}\right) u_{2}
\end{aligned}
$$

where $l$ is the length of the gripping arm $(3.9 \mathrm{~mm})$. Finally, the actuating comb-drive and sensing comb-drive are linearized with an input-output gain of $4.03 \mu \mathrm{N} V^{-1}$ and $1.87 \mu \mathrm{N} V^{-1}$ respectively.

Figure 3 depicts scanning electron microscope (SEM) images of the MEMS rotary micro-gripper fabricated on $25 \mu \mathrm{m}$ thick silicon using a commercial silicon-on-insulator MEMS foundry process (MEMSCAP) [28], where the zoomed-in image of the electrothermal sensor is illustrated in Fig. 3(c). The resistive sensors are made of curved silicon beams $(3 \mu \mathrm{m} \times 200 \mu \mathrm{m} \times 25 \mu \mathrm{m})$ and positioned adjacent to the heat sink plate with a $3 \mu \mathrm{m}$ gap. At room temperature, the resistance of each beam is measured to be $300 \Omega$. Time domain identification [29] is performed to obtain the dynamic behavior of each electrothermal displacement sensor. The sensors' step response has a time constant of $45 \mu \mathrm{s}$, which indicates a sensing bandwidth of $3.5 \mathrm{kHz}$. The sensitivity of the sensing system is determined by the chain rule:

$$
\frac{\partial V_{o 2}}{\partial u_{2}}=\frac{\partial V_{o 2}}{\partial x_{2}} \cdot \frac{\partial x_{2}}{\partial u_{2}}
$$

where the system gain is 1 , and the displacement is expressed as a quadratic function of comb-drive voltage in (5). As we include the electrostatic linearization given in (6b), the displacement can be described as a function of control signal $x_{2}=80.422 \times u_{2}$. Therefore, the sensitivity of the sensor

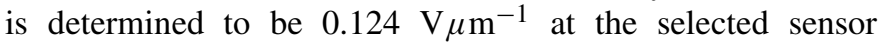
operating voltage $(9.65 \mathrm{~V})$. The temperature and resistance of the electrothermal sensor [30] are estimated to be $786{ }^{\circ} \mathrm{C}$ and $386 \Omega$ respectively. Although the sensor operates at high temperature, its relatively small size and geometric separation from the gripping arm limit the effect of thermal coupling to a sample during operation.
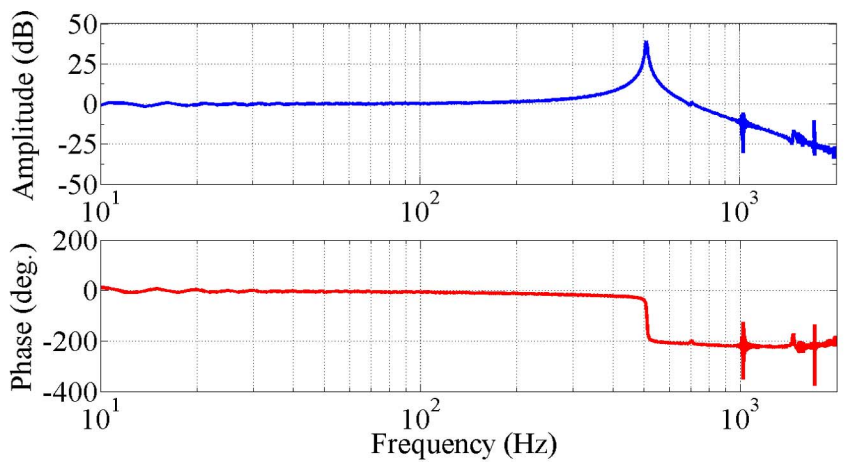

(a)
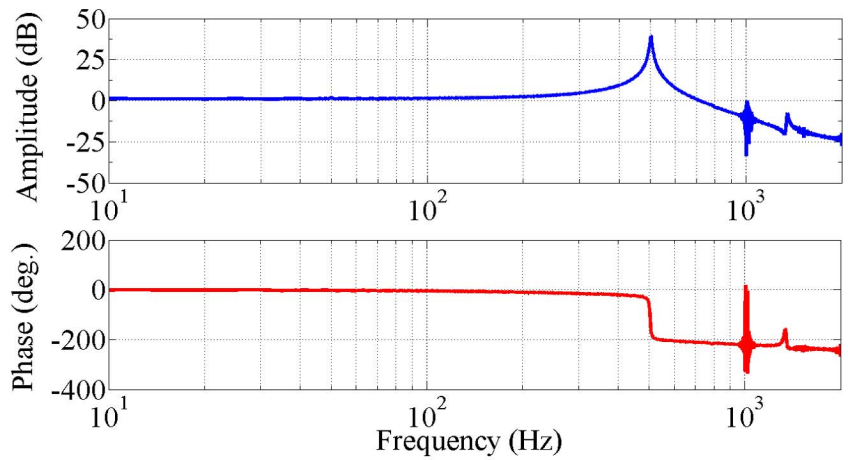

(b)

Fig. 4. Open loop frequency response of (a) the actuating system and (b) the sensing system at selected sensor bias voltage $(9.65 \mathrm{~V})$ up to $2 \mathrm{kHz}$.

\section{System IDENTIFICATION AND CALIBRATION OF FORCE SENSOR}

\section{A. System Identification}

To investigate the response of the actuating/sensing system to a driving voltage, the open-loop frequency analysis of the system is performed using a chirp signal. The frequency responses of the actuating and sensing systems are plotted in Fig. 4. It can be inferred from the figure that the systems are linear second-order systems with a resonance frequency at $f_{1}=502 \mathrm{~Hz}$ and $f_{2}=509 \mathrm{~Hz}$ and with damping ratio $\xi_{1}=\xi_{2}=5 \times 10^{-3}$. Since the electrothermal displacement sensor alone exhibits a linear characteristic within the frequencies of interest, the sensor is considered as a gain to the system, meaning that a second-order system is expected. Consequently, the transfer function of the actuating system $G_{1}$, and sensing system $G_{2}$ can be expressed as:

$$
\begin{aligned}
G_{1}(s) & =\frac{1.14}{s^{2}+31.54 s+9.94 \times 10^{6}} \\
G_{2}(s) & =\frac{1}{s^{2}+31.98 s+1.02 \times 10^{7}} .
\end{aligned}
$$

\section{B. Force Sensor Calibration}

In this work, experimental measurements are performed to obtain the value of $\beta$, which is the gain from the comb-drive voltage to the comb drive torque. The calibration technique used in this work allows us to identify this empirical relationship without using an external calibration device. This calibration method is used to calibrate the MEMS force sensor 


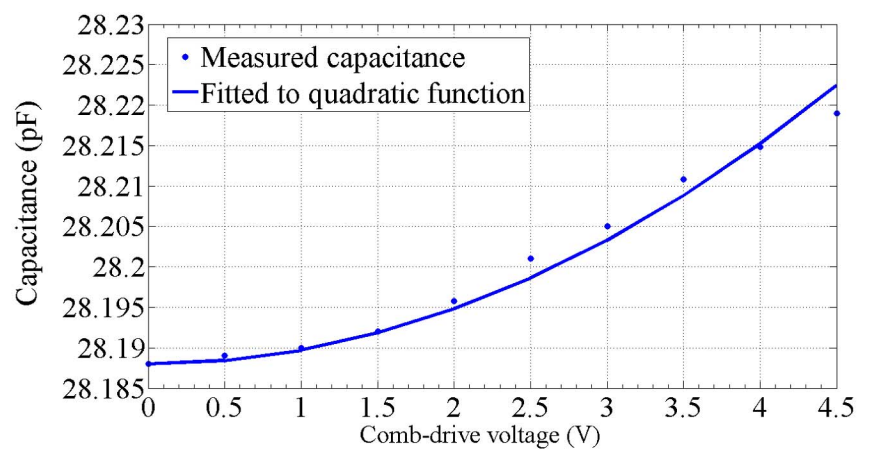

(a)

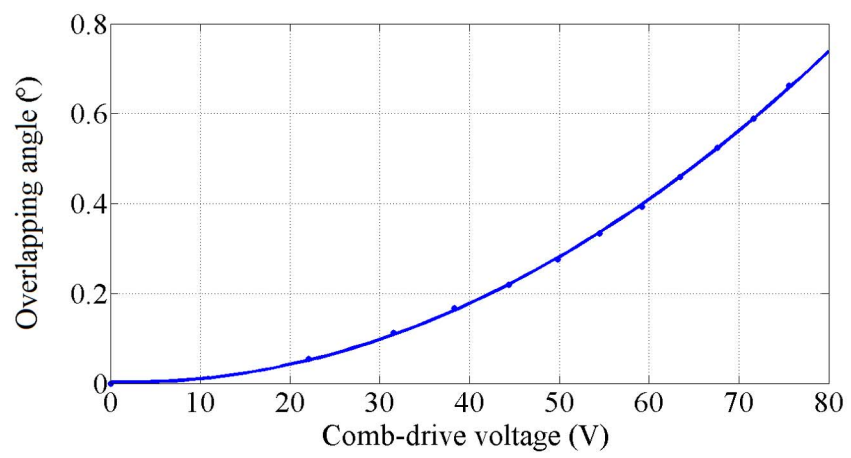

(b)

Fig. 5. The (a) capacitance and (b) angular displacement of the rotary combdrive are measured as a function of comb-drive voltage, using LCR meter and Micro System Analyzer respectively.

reported in [31] where the experimental result demonstrates the high accuracy of this calibration method.

Since the total capacitance of the comb-structure and angular displacement can be measured as a function of comb-drive voltage, the differential change in capacitance with respect to angular displacement is determined through the chain rule. Equations (10) and (11) describe the capacitance and the angular displacement as a function of $\beta$ and $\mathrm{V}$.

$$
\begin{aligned}
C & =\frac{2 \beta^{2}}{k_{\theta}} V^{2}+c_{p} \\
\beta V^{2} & =k_{\theta} \theta \rightarrow \theta=\left(\frac{\beta}{k_{\theta}}\right) V^{2}
\end{aligned}
$$

Fig. 5(a) plots the measured capacitance (in $\mathrm{pF}$ ) of the sensing comb structure versus comb-drive voltage, using an LCR meter. Through a curve fitting process, the capacitances can be estimated by a quadratic function as expressed in (12).

$$
C=1.7 \times 10^{-3} V_{2}^{2}+28.188
$$

Similarly, the angular displacement is measured in degrees using the MSA-400. The angular displacement is plotted as a function of comb-drive voltage in Fig. 5(b). The fitted quadratic function of the measured angular displacement is described in (13).

$$
\theta_{2}=1.15 \times 10^{-7} V_{2}^{2}
$$

By comparing the coefficients of (10) - (13), the electrostatic constant $\beta_{2}$, the angular stiffness $k_{\theta_{2}}$, and parasitic capacitance $c_{p}$ are experimentally identified to be

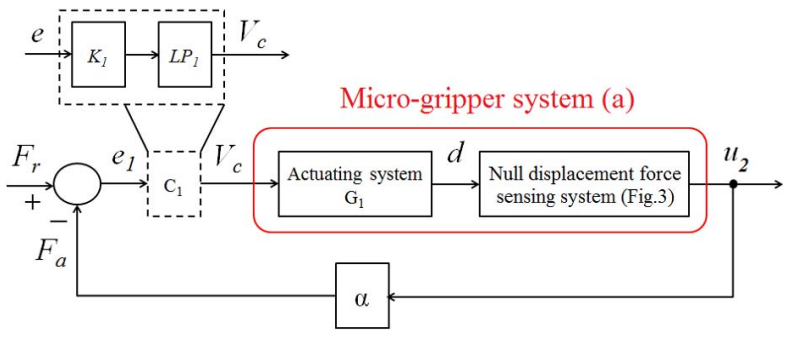

Fig. 6. Block diagram of null-displacement feedback force controlled MEMS rotary micro-gripper. The controller $\left(C_{1}\right)$ is designed to obtain satisfactory tracking performance.

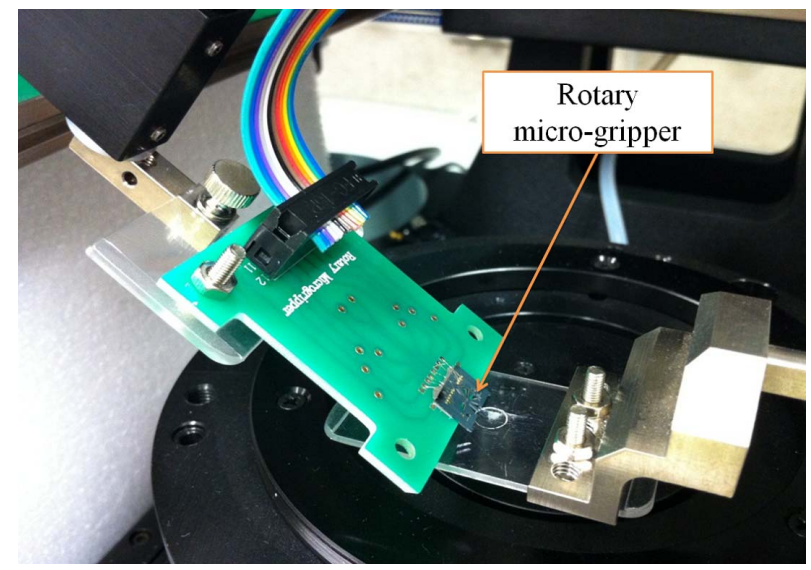

Fig. 7. Image of the fabricated rotary micro-gripper wire bonded to the PCB and positioned at an inclined angle, allowing the micro-gripper to access the sample.

$7.3913 \times 10^{-12} \mathrm{nN} \cdot \mathrm{mV}^{-2}, 64.3 \times 10^{-9} \mathrm{NmDeg}^{-1}$, and $28.188 \times$ $10^{-12} \mathrm{pF}$ respectively. As we include a function to linearize the electrostatic actuator as noted in (6b), the gain from the control signal $u_{2}$ to the comb drive torque $\tau_{2}$ is identified to be $7.613 \mathrm{nN} \cdot \mathrm{mV}^{-1}$. Since the micro-gripper is designed to rotate at a very small angle, and the gripping arm's length is measured to be $3.9 \mathrm{~mm}$, the gain from the control signal to the torque force developed at the gripping arm is identified to be $1.95 \mu \mathrm{NV}^{-1}$.

\section{Force CONTROL OF MEMS MiCRO-GRIPPER}

In this section, the force control scheme of the MEMS micro-gripper is discussed. First, we consider the complete micro-gripper system as a cascaded model comprising the actuating system followed by the null displacement force sensing system, where the input and the output of the system are the driving voltage and the contact force (measured as a function of the control signal $u$ ), respectively, as illustrated in Fig. 6(a). Integral controllers are implemented for the disturbance rejection and force control in this system where the block diagram of the entire null-displacement feedback force controlled MEMS rotary micro-gripper is illustrated in Fig. 6. The output (measured force) of the sensing system is used as a feedback signal to close the control loop. The empirical relationship between the force being measured and the control signal is assigned as a function $\alpha$, which was experimentally obtained and described in Section III.

$$
\alpha=1.95 \mu N V^{-1}
$$




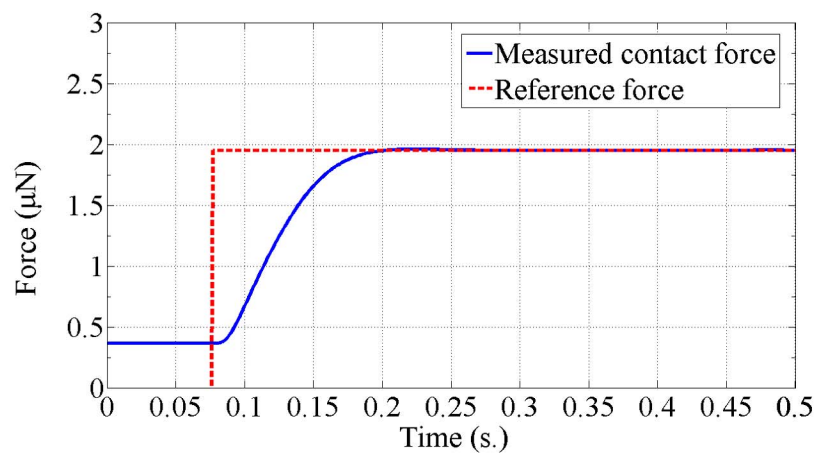

(a)

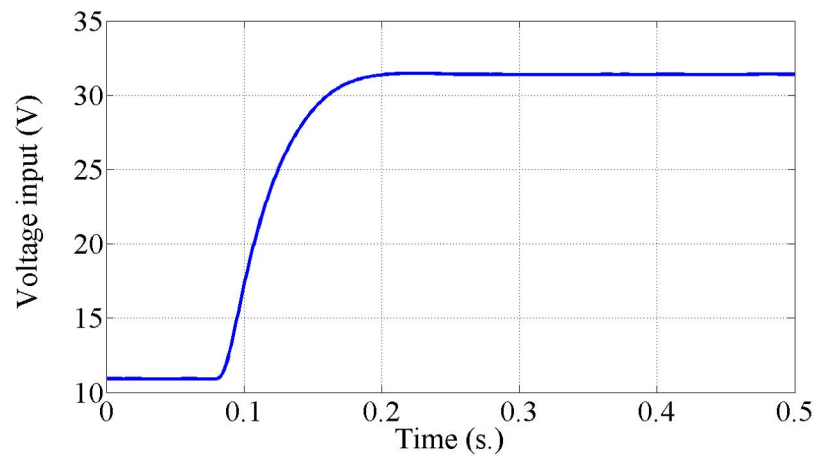

(c)

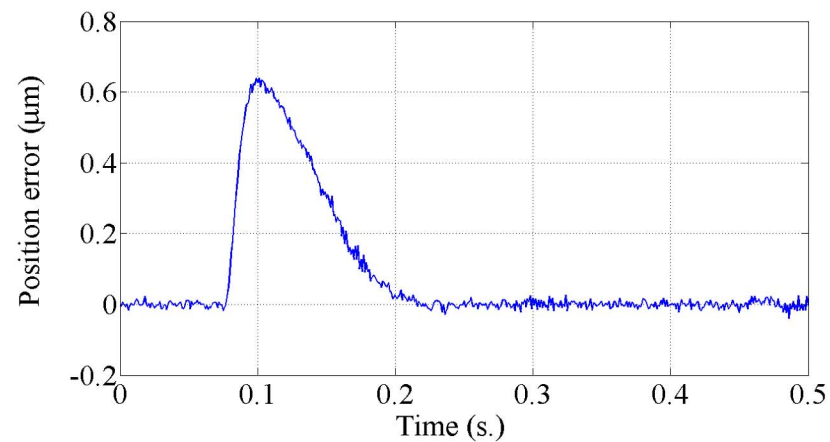

(b)

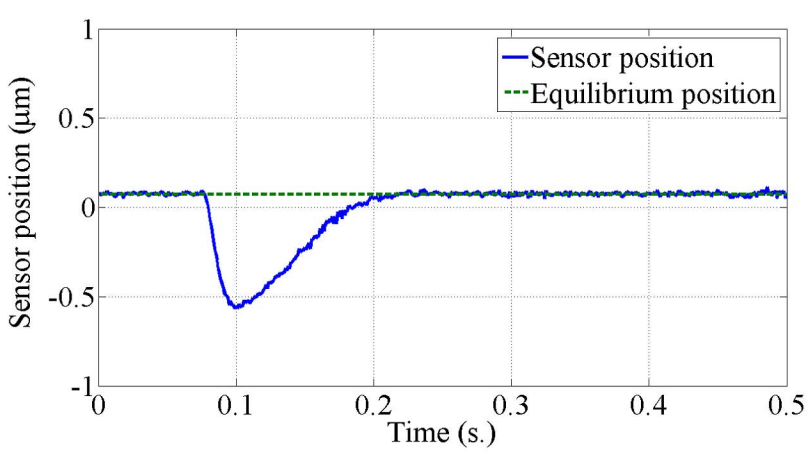

(d)

Fig. 8. Closed loop performance of the force controlled MEMS rotary micro-gripper performing constant reference force tracking, showing (a) reference and measured contact force, (b) error signal, (c) measured comb-drive voltage, and (d) sensor position.

The controller gains $\left(K_{1}=5, K_{2}=150\right)$ are experimentally tuned to achieve satisfactory force tracking and disturbance rejection. In order to suppress the dynamics of the gripper at its first resonance mode, a second order Butterworth filter is included as a part of the controller $\left(C_{1}, C_{2}\right)$. The lowpass filter attenuates the signal components whose frequencies are greater than the cutoff frequency of the gripping system $(500 \mathrm{~Hz})$. Due to the relatively low bandwidth requirements of the gripping operation, the corner frequency is selected at $100 \mathrm{~Hz}$, where it provides sufficient bandwidth to the gripping operation and suppresses higher frequency signals. The lowpass filter transfer functions included in the controller $C_{1}, C_{2}$ are described in (14).

$$
L P_{1}(s)=L P_{2}(s)=\frac{3.948 \times 10^{5}}{s^{2}+888.6 s+3.948 \times 10^{5}}
$$

\section{EXPERIMENTAL TESTING OF MICRO-GRIPPER}

In this study, a micromanipulation system is developed to verify the effectiveness of the micro-gripper design and the proposed control method. The fabricated rotary micro-gripper is wire-bonded to a PCB and mounted to the holder at an inclined angle as depected in Fig. 7. The position of the sample holder is adjusted beforehand so that it provides the best access to the sample. A dSPACE RT1103 board with ADC/DAC channels is used for processing the $\mathrm{I} / \mathrm{O}$ signals from/to the fabricated device, where these signals are then amplified by a voltage amplifier as required. The controller is implemented using MATLAB/Simulink software, where the contact force and gripping process are monitored in real time through a graphical user interface.

\section{A. Force Tracking Performance}

With the assumption that the micro-gripper is initially in contact with the sample, set-point force tracking is performed on a $40 \mu \mathrm{m}$ glass micro-bead to evaluate the effectiveness of the proposed controller. With selected gain $K_{1}=5$, the closed-loop response of the controlled micro-gripper to a step reference force of $1.95 \mu \mathrm{N}$ is plotted in Fig. 8(a). It can be seen from the figure that the contact force closely tracks the set point in steady state with a time constant of $31.8 \mathrm{~ms}$. The corresponding position error, applied comb-drive voltage, and the sensor position are also plotted in Fig. 8(b) - 8(d) respectively.

\section{B. Application}

A pick-and-place operation on a $60 \mu \mathrm{m}$ micro-bead is performed in free air while the contact force $F$ and combdrive voltage $V_{1}$ are measured and plotted in Fig. 9(a). The objective of this operation is to isolate a target micro-bead sample and relocate it away from the rest of the population. The manipulation process consists of four consecutive steps (I-IV). To begin with, the micro-gripper slowly approaches and makes contact with the sample as shown in Fig. 9(b) and 9(c) respectively. Note that while the initial contact force is very small $(0.25 \mu \mathrm{N})$, it is sufficient to effectively secure the sample between the gripping arms. Once the 

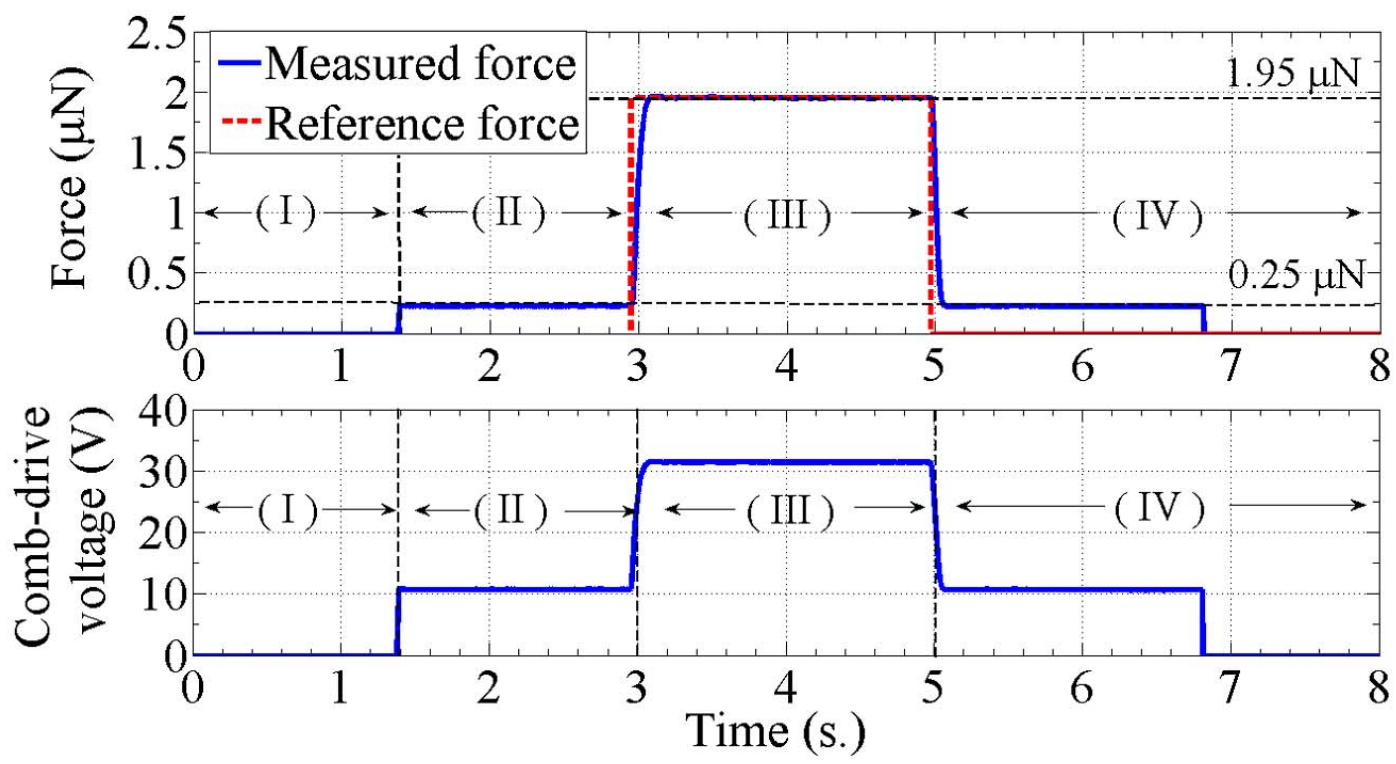

(a)

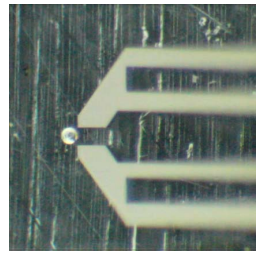

(b) I: Approaching

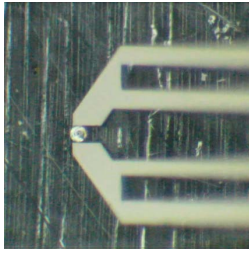

(c) II: Initial contact

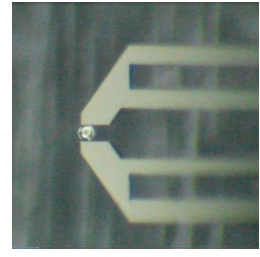

(d) III: Force exertion

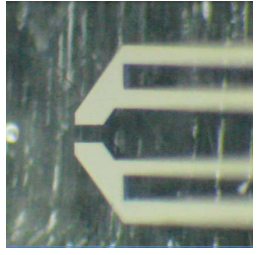

(e) IV: Release

Fig. 9. A pick-and-place operation on a $60 \mu \mathrm{m}$ micro-bead, performed in free air. (a) Time-domain plots of gripping force and comb-drive actuation voltage during the operation, and (b)-(e) images of the manipulation process.

reference force is defined, the micro-gripper applies a force to the sample, with the contact force accurately tracking the reference as shown in Fig. 9(d). Then, the micro-bead is relocated to a new location by manually adjusting the position of the micro-gripper's holder. At the end of the process (Fig. 9(e)), the object is released at the new location. The result demonstrates the satisfactory performance of the micro-gripper at handling a micro-bead with a gripping force of $1.95 \mu \mathrm{N}$ at a driving voltage of $31.5 \mathrm{~V}$, and relocating it to a desired position.

\section{CONCLUSION AND FUtURE WORK}

A force-controlled MEMS rotary micro-gripper and the development of a micromanipulation system are reported. The gripper produces large displacements at low operating voltages while the integrated electrothermal sensor allows the gripping force to be monitored during operation. The analysis of the micro-gripper structure and the experimental calibration of the electrothermal sensor are presented. A null displacement force sensing method is used for force measurement whose main advantage is that the dynamics of the sensing system have little influence on the measured force. Together with closedloop force control, the contact force exerted on the sample can be monitored and controlled in real-time, thus reducing the risk of damaging the sample and/or the device. The developed micromanipulation system is used to perform tests demonstrating set-point force tracking and a pick-and-place operation on $60 \mu \mathrm{m}$ micro-beads under the microscope. The experimental results indicate that the implemented controller provides excellent set-point force tracking with sufficiently fast time response.

One possibility for future work is to compare the accuracy of the force sensor with an external calibrated device to confirm the accuracy of the proposed method. Moreover, the implementation of more sophisticated vibration control techniques for the gripping arms. Considering that the grippers actuating/sensing systems have negative imaginary transfer functions, a strictly negative imaginary controller can be designed for vibration control [32]. This will allow the gripping process to be performed at higher speeds, creating the potential for fast, automated operation. Also, the application of a sensor fusion method is possible, as the gripper is equipped with both capacitive and electrothermal sensors [33]. The combination of the two sensing mechanisms may result in a better noise profile when the capacitive sensor operates at low frequencies and the electrothermal sensor operates at higher frequencies, as reported in [34] and [35]. Finally, an electrostatic drive selfsensing technique [36] can be implemented, where measurements of the current through the electrostatic actuator allow the drive to be simultaneously used for actuation and sensing. This allows the elimination of the existing electrothermal displacement sensors, further reducing the footprint of the micro-gripper. 


\section{REFERENCES}

[1] Y. Ansel, F. Schmitz, S. Kunz, H. P. Gruber, and G. Popovic, "Development of tools for handling and assembling microcomponents," J. Micromech. Microeng., vol. 12, no. 4, pp. 430-437, 2002.

[2] G. Thomell, M. Bexell, J.-A. Schweitz, and S. Johansson, "The design and fabrication of a gripping tool for micromanipulation," in Proc. 8th Int. Conf. Solid-State Sens. Actuators, vol. 2. Jun. 1995, pp. 388-391.

[3] T. Chen, L. Chen, L. Sun, and X. Li, "Electrostatically driven microgripper integrated piezoresistive force sensor," in Proc. Symp. Design, Test, Integr. Packag. MEMS/MOEM, Apr. 2008, pp. 376-380.

[4] T. C. Duc, G.-K. Lau, J. F. Creemer, and P. M. Sarro, "Electrothermal microgripper with large jaw displacement and integrated force sensors," J. Microelectromech. Syst., vol. 17, no. 6, pp. 1546-1555, Dec. 2008.

[5] D.-H. Kim, M. G. Lee, B. Kim, and Y. Sun, "A superelastic alloy microgripper with embedded electromagnetic actuators and piezoelectric force sensors: A numerical and experimental study," Smart Mater. Struct., vol. 14, no. 6, pp. 1265-1272, 2005.

[6] M. Kemper, "Development of a tactile low-cost microgripper with integrated force sensor," in Proc. IEEE Int. Conf. Control Appl., vol. 2. Sep. 2004, pp. 1461-1466.

[7] R.-J. Chang and C.-Y. Cheng, "Vision-based compliant-joint polymer force sensor integrated with microgripper for measuring gripping force," in Proc. IEEE/ASME Int. Conf. Adv. Intell. Mechatron., Jul. 2009, pp. $18-23$.

[8] H. Chang et al., "A rotary comb-actuated microgripper with a large displacement range," Microsyst. Technol., vol. 20, no. 1, pp. 119-126, 2014.

[9] F. Beyeler et al., "Monolithically fabricated microgripper with integrated force sensor for manipulating microobjects and biological cells aligned in an ultrasonic field," J. Microelectromech. Syst., vol. 16, no. 1, pp. 7-15, Feb. 2007.

[10] N. Ali, R. I. Shakoor, and M. M. Hassan, "Design, modeling and simulation of electrothermally actuated microgripper with integrated capacitive contact sensor," in Proc. IEEE 14th Int. Multitopic Conf. (INMIC), Dec. 2011, pp. 201-206.

[11] D. H. Wang, Q. Yang, and H. M. Dong, "A monolithic compliant piezoelectric-driven microgripper: Design, modeling, and testing," IEEE/ASME Trans. Mechatronics, vol. 18, no. 1, pp. 138-147, Feb. 2013

[12] D.-H. Kim, K. Kim, K.-Y. Kim, and S.-M. Cha, "Dexterous teleoperation for micro parts handling based on haptic/visual interface," in Proc. Int. Symp. Micromechatron. Human Sci. (MHS), Sep. 2001, pp. 211-217.

[13] S. Yu and B. J. Nelson, "Microrobotic cell injection," in Proc. IEEE Int. Conf. Robot. Autom., vol. 1. May 2001, pp. 620-625.

[14] M. Rakotondrabe and I. A. Ivan, "Development and force/position control of a new hybrid thermo-piezoelectric microgripper dedicated to micromanipulation tasks," IEEE Trans. Autom. Sci. Eng., vol. 8, no. 4, pp. 824-834, Oct. 2011.

[15] M. C. Carrozza, A. Eisinberg, A. Menciassi, D. Campolo, S. Micera, and P. Dario, "Towards a force-controlled microgripper for assembling biomedical microdevices," J. Micromech. Microeng., vol. 10, no. 2, pp. 271-276, 2000

[16] J. H. Kyung, B. G. Ko, Y. H. Ha, and G. J. Chung, "Design of a microgripper for micromanipulation of microcomponents using SMA wires and flexible hinges," Sens. Actuators A, Phys., vol. 141, no. 1, pp. 144-150, Jan. 2008.

[17] K. Kim, X. Liu, Y. Zhang, and Y. Sun, "Micronewton force-controlled manipulation of biomaterials using a monolithic MEMS microgripper with two-axis force feedback," in Proc. IEEE Int. Conf. Robot. Autom., May 2008, pp. 3100-3105.

[18] Y. Sun, S. N. Fry, D. P. Potasek, D. J. Bell, and B. J. Nelson, "Characterizing fruit fly flight behavior using a microforce sensor with a new comb-drive configuration," J. Microelectromech. Syst., vol. 14, no. 1, pp. 4-11, Feb. 2005.

[19] K. Kim, X. Liu, Y. Zhang, and Y. Sun, "Nanonewton force-controlled manipulation of biological cells using a monolithic MEMS microgripper with two-axis force feedback," J. Micromech. Microeng., vol. 18, no. 5, p. 055013, 2008.

[20] J. Park et al., "Identification and control of a sensorized microgripper for micromanipulation," IEEE/ASME Trans. Mechatronics, vol. 10, no. 5 , pp. 601-606, Oct. 2005.

[21] N. Chronis and L. P. Lee, "Electrothermally activated SU-8 microgripper for single cell manipulation in solution," J. Microelectromech. Syst., vol. 14, no. 4, pp. 857-863, Aug. 2005.
[22] H. Demaghsi, H. Mirzajani, and H. B. Ghavifekr, "A novel electrostatic based microgripper (cellgripper) integrated with contact sensor and equipped with vibrating system to release particles actively," Microsyst. Technol., vol. 20, no. 12, pp. 2191-2202, Dec. 2014.

[23] B. Piriyanont and S. O. Reza Moheimani, "MEMS rotary microgripper with integrated electrothermal force sensor," IEEE J. Microelectromech. Syst., vol. 23, no. 6, pp. 1249-1251, Dec. 2014.

[24] Y. Shen, E. Winder, N. Xi, C. A. Pomeroy, and U. C. Wejinya, "Closed-loop optimal control-enabled piezoelectric microforce sensors," IEEE/ASME Trans. Mechatronics, vol. 11, no. 4, pp. 420-427, Aug. 2006.

[25] K. H.-L. Chau, S. R. Lewis, Y. Zhao, R. T. Howe, S. F. Bart, and R. G. Marcheselli, "An integrated force-balanced capacitive accelerometer for low-g applications," in Proc. 8th Int. Conf. Solid-State Sens. Actuators, vol. 1. Jun. 1995, pp. 593-596.

[26] M. B. Coskun, S. Moore, S. O. R. Moheimani, A. Neild, and T. Alan, "Zero displacement microelectromechanical force sensor using feedback control," Appl. Phys. Lett., vol. 104, no. 15, pp. 153502-1-153502-4, 2014.

[27] C.-C. Tu, K. Fanchiang, and C.-H. Liu, " $1 \times$ N rotary vertical micromirror for optical switching applications," Proc. SPIE, vol. 5719, pp. 14-22, Apr. 2005.

[28] A. Cowen, G. Hames, D. Monk, S. Wilcenski, and B. Hardy, SOIMUMPs Design Handbook, Rev. 6.0, MEMSCAP Inc., Bernin, France, 2009.

[29] A. G. Fowler, A. Bazaei, and S. O. R. Moheimani, "Design and analysis of nonuniformly shaped heaters for improved MEMS-based electrothermal displacement sensing," J. Microelectromech. Syst., vol. 22, no. 3, pp. 687-694, Jun. 2013.

[30] A. Bazaei, Y. Zhu, R. Moheimani, and M. R. Yuce, "Analysis of nonlinear phenomena in a thermal micro-actuator with a built-in thermal position sensor," IEEE Sensors J., vol. 12, no. 6, pp. 1772-1784, Jun. 2012.

[31] S. I. Moore, M. B. Coskun, T. Alan, A. Neild, and S. O. R. Moheimani, "Feedback controlled MEMS force sensor for characterization of microcantilevers," IEEE J. Microelectromech. Syst., to be published.

[32] B. Bhikkaji, S. O. R. Moheimani, and I. R. Petersen, "A negative imaginary approach to modeling and control of a collocated structure," IEEE/ASME Trans. Mechatronics, vol. 17, no. 4, pp. 717-727, Aug. 2012.

[33] I. A. Mahmood, S. O. R. Moheimani, and K. Liu, "Tracking control of a nanopositioner using complementary sensors," IEEE Trans. Nanotechnol., vol. 8, no. 1, pp. 55-65, Jan. 2009.

[34] Y. Zhu, S. O. R. Moheimani, and M. R. Yuce, "Simultaneous capacitive and electrothermal position sensing in a micromachined nanopositioner," IEEE Electron Device Lett., vol. 32, no. 8, pp. 1146-1148, Aug. 2011.

[35] A. J. Fleming, A. G. Wills, and S. O. R. Moheimani, "Sensor fusion for improved control of piezoelectric tube scanners," IEEE Trans. Control Syst. Technol., vol. 16, no. 6, pp. 1265-1276, Nov. 2008.

[36] S. I. Moore and S. O. R. Moheimani, "Displacement measurement with a self-sensing MEMS electrostatic drive," J. Microelectromech. Syst., vol. 23 , no. 3, pp. 511-513, Jun. 2014

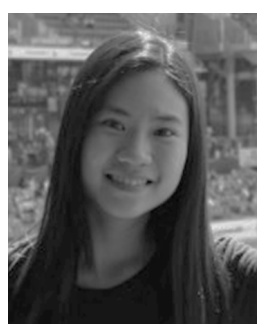

Busara Piriyanont (S'14) was born in Bangkok, Thailand. She received the B.Eng. (Hons.) degree in electrical engineering from Thammasat University, Bangkok, in 2008, and the M.E. degree in electrical engineering from the Toyohashi University of Technology, Toyohashi, Japan, in 2010. She received the Japanese Government Scholarship (MEXT) for the M.E. degree. She is currently pursuing the $\mathrm{Ph} . \mathrm{D}$. degree in electrical engineering with the University of Newcastle, Callaghan, NSW, Australia, where she was a recipient of the University of Newcastle International Post-Graduate Research Scholarships and the PostGraduate Research Scholarship. Her research interests include the design, analysis, and control of novel microelectromechanical systems-based microgripper for bio/micromanipulation applications. 


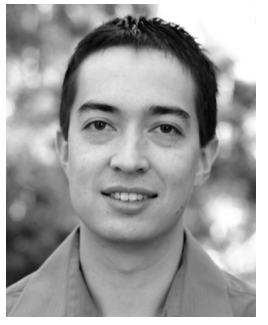

Anthony George Fowler (S'10-M'15) was born in Taree, NSW, Australia. He received the Bachelor's degree in electrical engineering and the Ph.D. degree in electrical engineering from the University of Newcastle, Callaghan, NSW, in 2010 and 2014, respectively, where he is currently a Post-Doctoral Fellow with the School of Electrical Engineering and Computer Science. His research interests include the design and analysis of novel microelectromechanical systems devices for energy harvesting and nanopositioning applications.

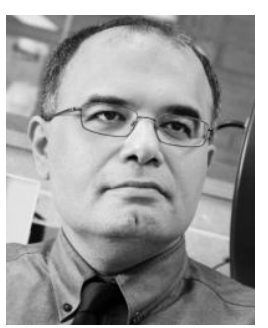

S. O. Reza Moheimani (F'11) received the Bachelor's degree in electrical engineering from Shiraz University, Shiraz, Iran, in 1991, and the Ph.D. degree from the University of New South Wales, Sydney, NSW, Australia, in 1996. In 1997, he joined the University of Newcastle, Callaghan, NSW, embarking on a new research program addressing the dynamics and control design issues related to high-precision mechatronic systems, where he is currently the Founder and Director of the Laboratory for Dynamics and Control of Nanosystems, a multimillion-dollar state-of-the-art research facility.
His current research interests are mainly in the area of ultrahighprecision mechatronic systems, with particular emphasis on dynamics and control at the nanometer scale, including applications of control and estimation in nanopositioning systems for high-speed scanning probe microscopy; modeling and control of microcantilever-based devices; control of microactuators in microelectromechanical systems; and design, modeling, and control of micromachined nanopositioners for on-chip atomic force microscopy.

Prof. Moheimani's work was recognized by a number of awards, including the International Federation of Automatic Control (IFAC) Nathaniel B. Nichols Medal (2014), the IFAC Mechatronic Systems Award (2013), the IEEE Control Systems Technology Award (2009), the Australian Research Council Future Fellowship (2009), the IEEE TRAns ACTIONS ON CONTROL Systems Technology Outstanding Paper Award (2007), the Australian Research Council Post-Doctoral Fellowship (1999), and several best student paper awards in various conferences. He has served on the Editorial Boards of a number of journals, including the IEEE TRANSACTIONS ON MEChatRonics/ASME Transactions on Mechatronics, the IEEE TRANSactions on Control Systems Technology, and Control Engineering Practice. He has chaired several international conferences and workshops, and is currently the Chair of the IFAC Technical Committee on Mechatronic Systems. He has authored over 300 refereed papers, and five books and edited volumes. $\mathrm{He}$ is a Fellow of IFAC and the Institute of Physics, U.K. 